NASA

Technical Paper 1566
AVRADCOM

Technical Report 80-A-1

\title{
On the Nonlinear Deformation Geometry of Euler-Bernoulli Beams
}

Dewey H. Hodges and Robert A. Ormiston Aeromechanics Laboratory AVRADCOM Research and Technology Laboratories Ames Research Center Moffett Field, California

David A. Peters Washington University

St. Louis, Missouri

\section{N/SA}

National Aeronautics and Space Administration

Scientific and Technical Information Office

1980 